\title{
Spin-Flipping Polarized Deuterons At COSY a
}

\author{
K. Yonehara*, A.D. Krisch*, V.S. Morozov*, R.S. Raymond*, V.K. Wong*, \\ U. Bechstedt ${ }^{\dagger}$, R. Gebel ${ }^{\dagger}$, A. Lehrach ${ }^{\dagger}$, B. Lorenz ${ }^{\dagger}$, R. Maier ${ }^{\dagger}$, D. Prasuhn ${ }^{\dagger}$, \\ A. Schnase ${ }^{\dagger}$, H. Stockhorst ${ }^{\dagger}$, D. Eversheim** ${ }^{* *}$ F. Hinterberger ${ }^{* *}$, \\ H. Rohdjess ${ }^{* *}$, K. Ulbrich ${ }^{* *}$ and W. Scobel ${ }^{*}$ \\ *Spin Physics Center, University of Michigan, Ann Arbor, MI 48109-1120, USA \\ ${ }^{\dagger}$ Forschungszentrum Jïlich, Inst. für Kernphysik, Postfach 1913, D-52425 Jïlich, Germany \\ ${ }^{* *}$ Helmholtz-Inst. für Strahlen- und Kernphysik, Univ. Bonn, D-53115 Bonn, Germany \\ ${ }^{\ddagger}$ Inst. fiir Exper-physik, Univ. Hamburg, Luruper Chaussee 149, D-22761 Hamburg, Germany
}

\begin{abstract}
We recently stored a $1.85 \mathrm{GeV} / \mathrm{c}$ vertically polarized deuteron beam in the COSY Ring in Jülich; we then spin-flipped it by ramping a new air-core rf dipole's frequency through an rfinduced spin resonance to manipulate the polarization direction of the deuteron beam. We first experimentally determined the resonance's frequency and set the dipole's $\mathrm{rf}$ voltage to its maximum; then we varied its frequency ramp time and frequency range. We used the EDDA detector to measure the vector and tensor polarization asymmetries. We have not yet extracted the deuteron's tensor polarization spin-fiip parameters from the measured data, since our short run did not provide adequate tensor analyzing-power data at $1.85 \mathrm{GeV} / \mathrm{c}$. However, with a $100 \mathrm{~Hz}$ frequency ramp and our longest ramp time of $400 \mathrm{~s}$, the deuterons' vector polarization spin-flip efficiency was $48 \pm 1 \%$.
\end{abstract}

Recently polarized deuteron experiments have become an accessible and interesting area of subatomic physics. Studying the spin-flipping and polarization dynamics of polarized deuteron beams is an important step towards polarized deuteron, and thus polarized neutron, scattering experiments. Many polarized scattering experiments require frequent spin-direction reversals (spin-flips), while the polarized beam is stored, to reduce their systematic errors. Earlier we used an rf solenoid to study, for first time, spin flipping and spin manipulation of a simultaneously vector and tensor polarized $270 \mathrm{MeV}$ deuteron beam stored in the IUCF Cooler Ring and obtained rather interesting results [1]. We recently studied spin flipping with an if dipole of a simultaneously vector and tensor polarized $1.85 \mathrm{GeV} / \mathrm{c}$ deuteron beam at COSY's higher energy.

In any flat circular accelerator or storage ring, each deuteron's spin precesses around the Stable Spin Direction (SSD), which is defined by the ring's magnetic structure. With no horizontal magnetic fields in the ring, the SSD points along the vertical fields of the ring's bending magnets. The spin tune $v_{s}$, which is the number of spin precessions during one turn around the ring, is proportional to the deuteron's energy

$$
v_{s}=G \gamma
$$

\footnotetext{
a Supported by research grants from the U.S. Department of Energy and the German BMBF Ministry.
} 
where $G=(g-2) / 2=-0.1426$ is the deuteron's gyromagnetic anomaly and $\gamma$ is its Lorentz energy factor.

The polarization can be perturbed by the horizontal if magnetic field from either an rf-solenoid or an rf-dipole. This perturbation can induce an $\mathrm{rf}$ spin resonance, which can flip the spin of the stored polarized deuterons; the resonance's frequency is

$$
f_{r}=f_{c}\left(k \pm v_{s}\right),
$$

where $f_{c}$ is the deuteron's circulation frequency and $k$ is an integer. Sweeping the rf magnet's frequency through $f_{r}$ can flip the Ring's SSD [2]. For an adiabatic frequency sweep, each deuteron's vector polarization follows the SSD as it rotates by $180^{\circ}$; this causes a vector spin flip. The modified [3] Froissart-Stora formula [4] relates the beam's initial vector polarization $P_{i}$ to its final polarization after crossing the resonance $P_{f}$

$$
P_{f}=P_{i}\left\{(1+\eta) \exp \left[-\frac{\left(\pi \varepsilon f_{c}\right)^{2}}{\Delta f / \Delta t}\right]-\eta\right\}
$$

where $\eta$ is the spin-flip efficiency, $\varepsilon$ is the resonance strength, and $\Delta f / \Delta t$ is the resonance crossing rate, while $\Delta f$ is the ramp's frequency range during the ramp time $\Delta t$.

In addition to the three ordinary vector polarization components, the polarization of a beam of spin-1 particles is also usually described by its tensor polarization. Unfortunately, there was no analyzing power data available at $1.85 \mathrm{GeV} / \mathrm{c}$; thus, we were unable to extract the deuteron's tensor polarization from the measured asymmetries. Thus, we will only present results on spin-flipping the deuteron vector polarization. In future runs we plan to measure the analyzing powers and thus obtain the tensor polarization.

The apparatus used for this experiment is shown in Fig. 1; it includes the COSY storage ring [5], the EDDA detector, the Low Energy Polarimeter, the injector Cyclotron, the polarized ion source, and the rf dipole, which was two 6-turn air-core copper coils, installed around a fast quadrupole's ceramic chamber. As part of an LC resonant circuit, the of dipole normally ran at about $4.3 \mathrm{kV}$ rms producing an $\int B d l$

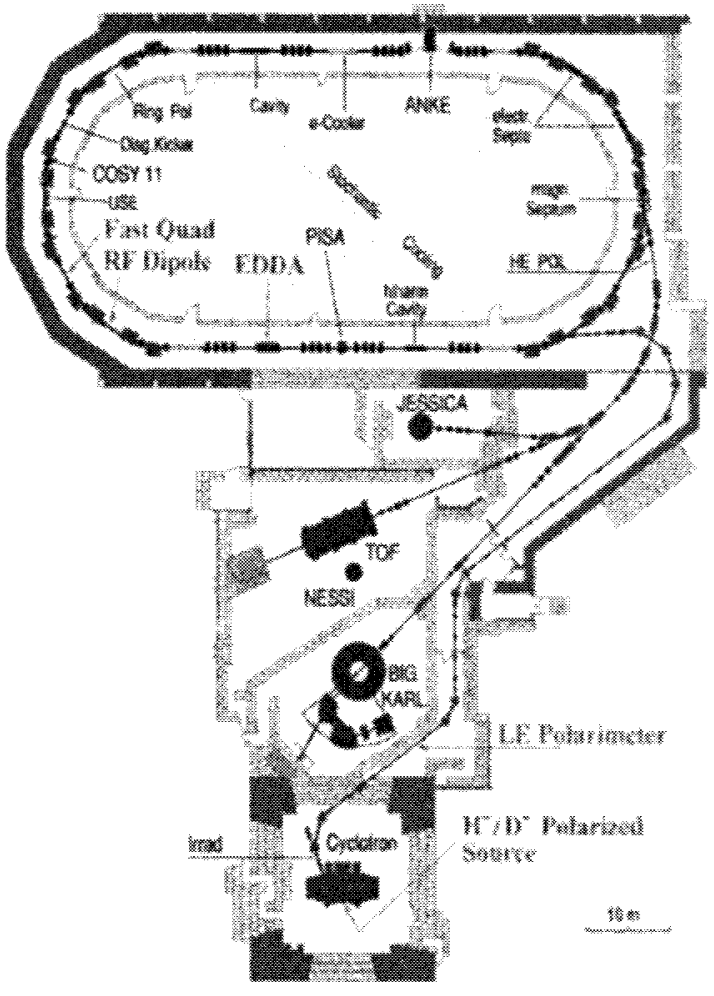

FIGURE 1. Layout of the COSY Storage Ring, with its injector Cyclotron, polarized ion source, rf dipole, EDDA detector, and Low Energy Polarimeter. 
of about $0.15 \mathrm{~T} \cdot \mathrm{mm}$ rms. The beam emerging from the polarized $\mathrm{D}^{-}$ion source was accelerated by the Cyclotron to COSY's injection energy. The Low Energy Polarimeter (LEP), which was between the Cyclotron and COSY, monitored the beam's polarization before injection into COSY. The LEP data was mostly used to check the stable operation of the ion source and Cyclotron.

To reduce our systematic errors, we cycled the polarized $\mathrm{D}^{-}$source through five polarization states with nominal vector polarization values of $0,-2 / 3,-1 / 3,-1,+1$. Although no analyzing power data were available at $1.85 \mathrm{GeV} / \mathrm{c}$, we measured the deuteron scattering asymmetries in the EDDA detector, which are related to the vector and tensor polarizations.

We first determined the spin resonance's approximate location $f_{r}=f_{c}\left(1-\left|v_{s}\right|\right)$ by ramping the rf dipole's frequency through $\Delta f=100 \mathrm{~Hz}$ around the calculated frequency of $f_{r}=917.4 \mathrm{kHz}$; we then continued with $100 \mathrm{~Hz}$ ramps next to each previous frequency range until the beam was partly depolarized, as shown in Fig. 2. The source was cycled through only the +1 and -1 vector polarized states; after each $100 \mathrm{~Hz}$ ramp of duration $\Delta t=19 \mathrm{~s}$, we measured the deuteron's left-right scattering asymmetry, which was proportional to its vector polarization. Fig. 2 shows the ratio $P_{f} / P_{i}$ of the asymmetries measured before $\left(P_{i}\right)$ and after $\left(P_{f}\right)$ ramping the rf dipole. These data show that the resonance was at about $916.85 \mathrm{kHz}$, which is near the calculated $f_{r}$.

We then mapped the spin resonance by measuring the asymmetry ratio, with the rf dipole at different fixed frequencies near $916.85 \mathrm{kHz}$. These data, plotted in Fig. 3, have a wide and shallow dip centered near $916.85 \mathrm{kHz}$; we fit this data to a second-order Lorentzian. This wide and shallow shape could be due to a combination of a somewhat weak dipole and a finite spin tune spread.

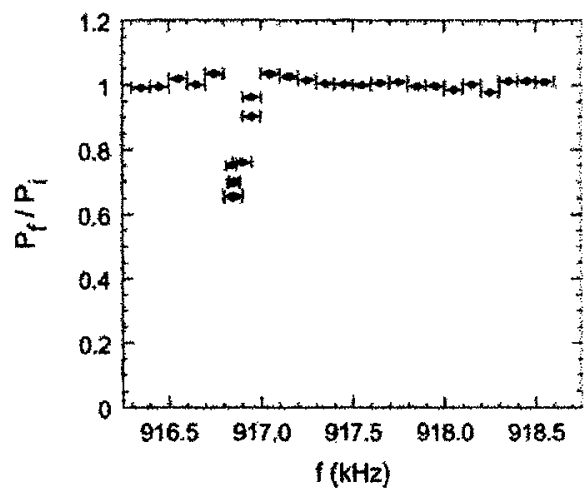

FIGURE 2. The measured deuteron's final-toinitial vector polarization ratio at $1.85 \mathrm{GeV} / \mathrm{c}$ is plotted against the range of each frequency ramp; a horizontal bar shows each ramp's $\Delta f$ range.

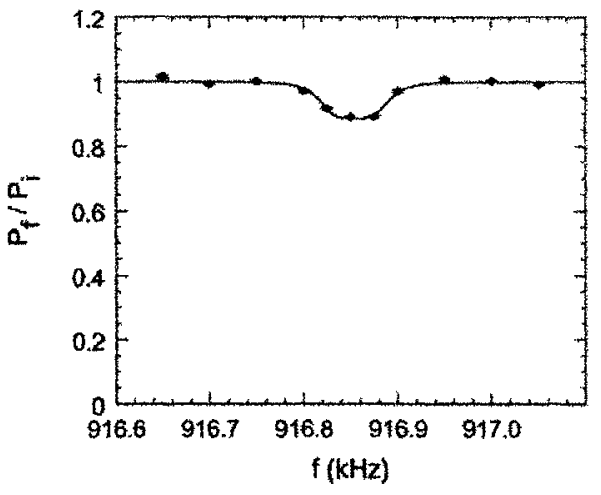

FIGURE 3. The measured deuteron's final-toinitial vector polarization ratio at $1.85 \mathrm{GeV} / \mathrm{c}$ is plotted against the rf-dipole's fixed frequency. The curve is a second-order Lorentzian fit.

After setting the rf dipole's voltage to its maximum and keeping the frequency range fixed at $\Delta f=100 \mathrm{~Hz}$, we spin-flipped the beam by varying the frequency ramp time $\Delta t$. The measured left-right scattering asymmetries for the five polarization states are plotted in Fig. 4; the curve for each polarization state is a fit to Eq. (3). Notice that all five curves cross at the same non-zero point near $100 \mathrm{~s}$. This indicates that, after 
subtracting the systematic offset of about $1.5 \%$, each asymmetry was indeed linearly proportional to the state's vector polarization. Fig. 4 shows that the vector polarization was partially spin-flipped for the two longest ramp times of 200 and $400 \mathrm{~s}$.

To further analyze the data, we first subtracted the unpolarized offset, shown in Fig. 4 by a solid line, from each other plotted asymmetry; we next divided the result by each corresponding initial asymmetry. Then, for each $\Delta t$, we averaged the four ratios and plotted the average $P_{f} / P_{i}$ ratio in Fig. 5 vs. $\Delta t$; these data were fit to Eq. (3), which gave a spin-flip efficiency $\eta=48 \pm 1 \%$.

Fig. 5 suggests that increasing the ramp time even further would probably not significantly increase the spin-flip efficiency. Probably the $\Delta f=100 \mathrm{~Hz}$ frequency range did not cover the full resonance width, which limited the maximum spin-flip efficiency. We plan to increase the deuteron spin-flip efficiency by increasing the rf-dipole's strength, perhaps by increasing its input power and building a ferrite box around it.

We would like to thank the entire COSY staff for the successful operation of the Cooler Synchrotron, the injector Cyclotron, and the polarized ion source.

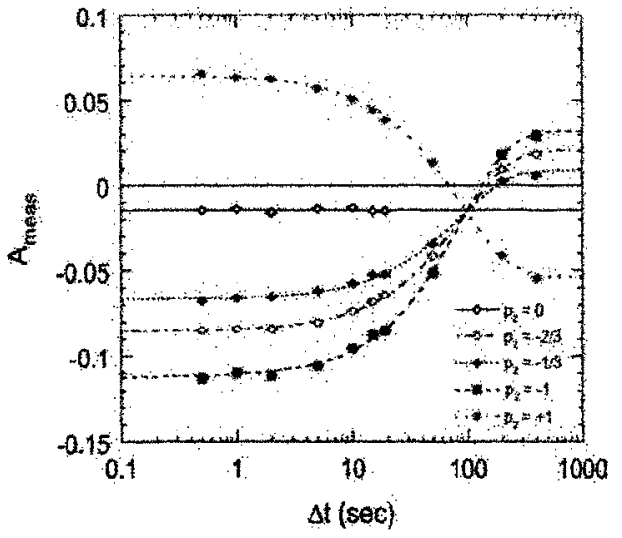

FIGURE 4. The measured deuterons' left-right scattering asymmetry for five polarization states at I.85 GeV/c is plotted against the rf-dipole's ramp time $\Delta t$. The curves are fits using Eq. (3).

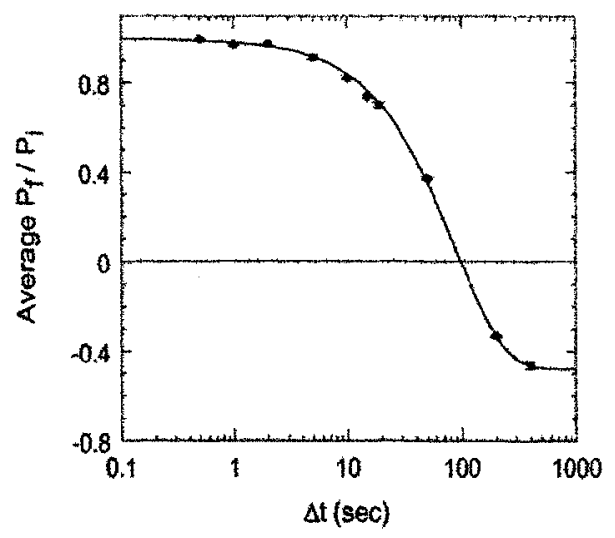

FIGURE 5. The $1.85 \mathrm{GeV} / \mathrm{c}$ deuterons' final-toinitial vector polarization ratio, averaged for the 4 polarized states, is plotted vs. the rf-dipole's ramp time $\Delta t$. The curve is a fit to Eq. (3).

\section{REFERENCES}

1. V.S. Morozov et al., "Spin flipping of a $270 \mathrm{MeV}$ deuteron beam", submitted to Phys.Rev. Lett.

2. B.B. Blinov et al., Phys. Rev. Lett., 88, 014801 (2002).

3. V.S. Morozov et al., Phys. Rev. ST-AB 4, 104002 (2001).

4. M. Froissart and R. Stora, Nucl. Instrum. Meth. 7, 297 (1960).

5. R. Maier, Nucl. Instrum. Meth. A390, 1-8 (1997). 